\section{Design and fabrication of enhanced polymer waveguide platforms for absorption-based optical chemical sensors}

To cite this article: C S Burke et al 2004 Meas. Sci. Technol. 151140

View the article online for updates and enhancements.
Related content

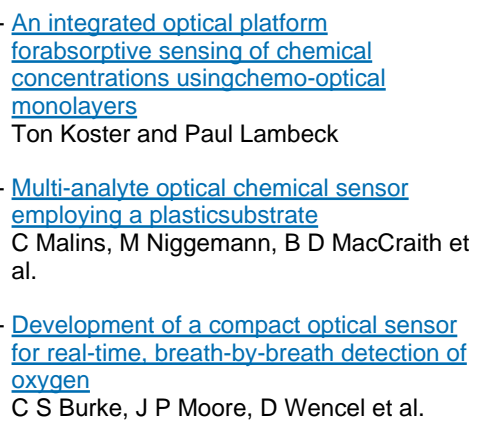

Development of a compact optical sensor for real-time, breath-by-breath detection of oxygen

C S Burke, J P Moore, D Wencel et al.

\footnotetext{
Recent citations
} 


\title{
Design and fabrication of enhanced polymer waveguide platforms for absorption-based optical chemical sensors
}

\author{
C S Burke ${ }^{1}$, L Polerecky ${ }^{2}$ and B D MacCraith ${ }^{1}$ \\ ${ }^{1}$ National Centre for Sensor Research, School of Physical Sciences, Dublin City University, \\ Glasnevin, Dublin 9, Ireland \\ ${ }^{2}$ Microsensors Group, Max Planck Institute for Marine Microbiology, Celsiusstrasse 1, \\ 28359 Bremen, Germany \\ E-mail: Brian.MacCraith@dcu.ie
}

Received 23 February 2004

Published 13 May 2004

Online at stacks.iop.org/MST/15/1140

DOI: $10.1088 / 0957-0233 / 15 / 6 / 014$

\begin{abstract}
The development of mass-producible polymer waveguide chips as enhanced platforms for absorption-based optical chemical sensors employing thin colorimetric films is reported here. The chip design is based on a previously reported theoretical study relating to the optimization of the sensitivity of optical absorption-based sensors employing a single reflection configuration. Here, the theoretical analysis is extended to a multiple reflection configuration in order to determine the dependence of sensitivity on interaction length, and the findings are investigated experimentally. The existence of the optimum sensing conditions predicted by theory is demonstrated empirically by examining the $\mathrm{pH}$ response of polymer waveguide platforms coated with a sol-gel derived sensing layer doped with a colorimetric $\mathrm{pH}$ indicator. This work has major implications for the fabrication of miniaturized, disposable sensor platforms that demonstrate enhanced sensitivity when compared with those utilizing more commonly employed optical configurations.
\end{abstract}

Keywords: solution $\mathrm{pH}$, polymer waveguide platforms, absorption-based optical sensing

\section{Background}

Absorption-based optical chemical sensors based on the colour change of a thin sensing film have been applied to the detection of various analytes including $\mathrm{NH}_{3}$ [1-4], $\mathrm{CO}_{2}$ [5-7], chlorine [8], water vapour [9-11] and $\mathrm{pH}[12-15]$. The use of a thin film is attractive as it reduces sensor response time, can be more easily incorporated into miniaturized sensor systems via a variety of coating procedures and requires less material for its fabrication when compared to bulk systems. This latter feature is important when dealing with expensive reagents or dyes for which synthesis is complicated or lengthy. Various optical configurations employing thin sensor films have been reported, including reflectance [16, 17], transmittance $[3,11,18]$ and both fibre and planar waveguide-based devices employing evanescent-wave interrogation of the sensing layer $[8,9]$. Planar waveguides offer several advantages over fibrebased systems. They can be coated with a thin film using a variety of techniques including dip coating and spin coating. Often, these techniques are either impractical or more difficult to implement in the case of optical fibres. Planar waveguides are inherently more robust due to their geometry and can be easily integrated into microfluidic systems for lab-on-a-chip applications.

The 'active waveguide' configuration demonstrated by Hisamoto and Suzuki [19] shows a marked improvement in sensitivity over devices operating in an evanescent-wave configuration as does the work of Puyol et al [20], which is based on 'integrated waveguide absorbance optodes' (IWAOs). However, both configurations suffer from a high degree of 
Enhanced polymer waveguide platforms for absorption-based sensors

complexity in their fabrication process. In the case of the 'active waveguide' device, both sputtering and spin-coating techniques were employed to produce a device that made use of four different materials. The relatively complex nature of this design along with the use of prism couplers renders this device impractical from the point of view of both mass production and field use. The IWAOs developed by Puyol et al employ input and output anti-resonant reflecting optical waveguide (ARROW) structures and a free propagation region between the two where the sensing membrane is placed. The membrane supports the guided mode from the input waveguide and this mode is then coupled to the larger output waveguide for detection. The fabrication of this device is again relatively complex, involving low-pressure chemical vapour deposition, plasma-enhanced chemical vapour deposition, reactive ion etching and spin-coating steps. There is a clear need for much simpler approaches which would be compatible with mass production and sufficiently robust for deployment outside the laboratory.

In terms of sensor performance however, sensitivity is one of the key issues. Recent work has highlighted an optimization strategy for absorption-based optical chemical sensors based on a rigorous electromagnetic wave analysis of platform sensitivity and its dependence on optical configuration [21]. The analysis was developed in the context of multimode waveguide platforms and indicated that a significant enhancement in sensitivity could be achieved by tailoring the angle of incidence of the interrogating light on the sensing layer. These predictions were verified experimentally and applied to the fabrication of a LED-based prototype sensor incorporating a single-reflection configuration. This prototype demonstrated a pronounced sensitivity enhancement when compared to configurations employing evanescent-wave interrogation and highlighted the strength of the optimization procedure. We now report a significant extension of these principles to enable fabrication of mass-producible sensor platforms with enhanced sensitivity. First, we examine the implications of extending the interrogation of the sensing layer from a single reflection to multiple reflections and establish an optimization strategy which enables identification of the conditions for which sensitivity is maximized. Second, we describe the design and fabrication of a generic polymer waveguide platform which exploits these principles and which is compatible with low-cost mass production. Finally, in order to prove these principles, we demonstrate a $\mathrm{pH}$ sensor system using a sol-gel derived film (doped with a pH indicator) which is coated on the planar waveguide platform.

\subsection{Theoretical model}

Figure 1 is a generalized representation of the platform considered in the optimization procedure reported earlier [21]. It consists of an analyte-sensitive, colorimetric thin film of refractive index $n_{s}$ coated on a planar substrate with refractive index $n_{g}$. The analyte under investigation is present in the superstrate environment (refractive index $n_{e}$ ) and diffuses into the sensing layer where it causes a change in the absorption coefficient, $\alpha_{s}$, of the layer. The magnitude of the change in $\alpha_{s}$ is dependent on analyte concentration. Light from a source that is spectrally matched to the absorption maximum of the

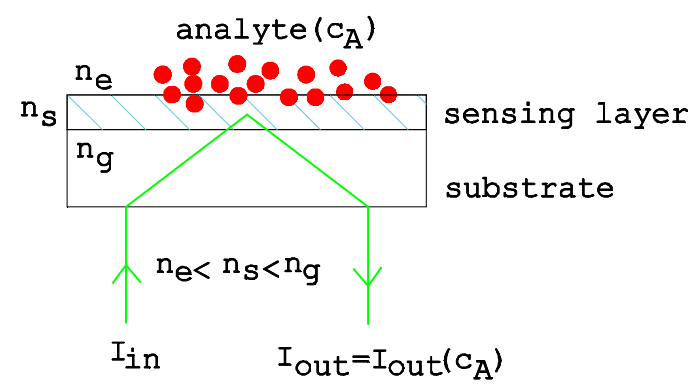

Figure 1. Schematic of sensor configuration reported in [21].

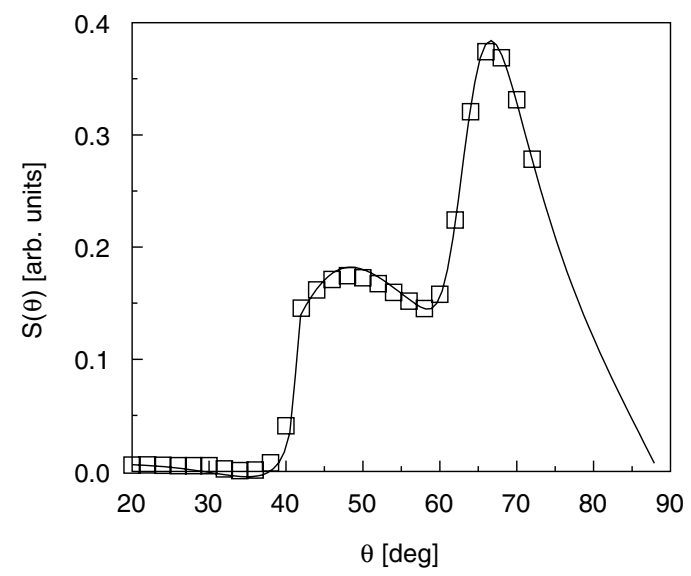

Figure 2. Dependence of sensitivity on angle of interrogation in a single reflection configuration. Squares represent experimental data [21].

sensing layer is coupled into the substrate and interrogates the layer, after which it is outcoupled to an appropriate detector where its intensity is measured. Changes in analyte concentration, and hence $\alpha_{s}$, result in a modulation of the light intensity which is detected at the output of the platform, thereby providing the basis for the sensor.

For the purposes of the theoretical analysis, the following refractive index condition is assumed:

$$
n_{e}<n_{s}<n_{g} .
$$

The sensitivity of the device is defined as

$$
S\left(c_{A}\right)=\mathrm{d} V / \mathrm{d} c_{A}
$$

where $V$ is the output signal measured by the detector and $c_{A}$ is the analyte concentration. It is possible to quantify the variation of output signal with respect to the different optical parameters of the system by analysing the dependence of reflectivity $(R)$ on these parameters. In particular, the sensitivity, $S$, was calculated as a function of the angle, $\theta$, at which the light interrogates the sensing layer. The results are shown in figure 2 where the sensitivity is plotted against angle of incidence on the sensing layer. There is clearly an optimum angle, $\theta_{\mathrm{opt}}$, at which sensitivity is maximum and the existence of this peak was verified experimentally, as indicated by the data points represented as squares in figure 2 . In this situation, the guiding layer consisted of glass with refractive index $n_{g}=1.515$. The sensing layer was a sol-gel derived thin film with refractive index $n_{s}=1.43$, and the environment 
(air) had a refractive index, $n_{e}=1.00$. The thickness of the sensing layer was $t_{s}=400 \mathrm{~nm}$, as measured by profilometry and the wavelength of the interrogating source was $\lambda=$ $543.5 \mathrm{~nm}$ corresponding to the green $\mathrm{He}-\mathrm{Ne}$ laser used. These data were reported previously in [21].

In order to predict the effect of multiple reflections on sensitivity for a range of sensing layer absorption coefficients, $\alpha_{s}$, and interaction lengths, $L_{\text {int }}$ (this being the length of sensing layer interrogated by the light), we have extended our theoretical analysis. The output signal, $V$, is dependent on several parameters including the input power of the light source, coupling efficiencies of the sensor head (i.e. the fraction of light coupled into and out of the platform) and the efficiency with which the optical signal is converted to an electronic signal by the sensor electronics. It is assumed here that none of these parameters are influenced by changes in $\alpha_{s}$, and therefore analyte concentration $\left(c_{A}\right)$, as they are not dependent on the properties of the sensing layer. The output signal can, therefore, be written as

$$
V=\Gamma \mathcal{T}\left(c_{A}, L_{\text {int }}\right)+V_{0}
$$

where $\Gamma$ is that element of the signal that is independent of $c_{A}, V_{0}$ is a signal offset introduced by the electronics and $\mathcal{T}$ is the attenuation factor of the sensor platform. $\mathcal{T}$ is dependent on both $c_{A}$ and the interaction length, $L_{\text {int }}$. Substituting equation (3) into equation (2), we can define the platform sensitivity as follows:

$$
S\left(c_{A}\right)=\Gamma \frac{\mathrm{d} \mathcal{T}\left(c_{A}, L_{\mathrm{int}}\right)}{\mathrm{d} c_{A}} .
$$

It can be shown that

$$
S\left(c_{A}\right)=-\Gamma \xi L_{\text {int }} \tilde{S}_{\left(\alpha_{s}\right)} \exp \left[-\xi L_{\text {int }}\right]
$$

where the relative sensitivity, $\tilde{S}_{\alpha_{S}}$, of the sensing layer is given by

$$
\tilde{S}_{\alpha_{s}}=\frac{1}{\alpha_{s}} \frac{\partial \alpha_{s}}{\partial c_{A}}
$$

and the parameter $\xi$ is directly proportional to $\alpha_{s}$ for low values of $\alpha_{s}$.

From the presence of the term $\xi L_{\text {int }}$ in equation (5), it is clear that the sensitivity of the platform increases with interaction length provided the argument of the exponential term remains sufficiently small. This is due to the fact that, for low values of $\xi L_{\text {int }}$, the exponential term is approximately equal to 1. However, the model summarized in equation (5) also shows that sensitivity will begin to decrease exponentially given a sufficiently long interaction length or sufficiently high absorption coefficient. These findings are illustrated in figure 3, which shows the relationship between sensitivity and interaction length for various extinction coefficients of the sensing layer when the interrogating light is incident on the layer at the optimum angle of incidence, $\theta_{\mathrm{opt}}$, determined from the theoretical analysis [22]. In this case, $t_{s}=800 \mathrm{~nm}$ and the values of all other optical parameters remain unchanged from those used to generate the results shown in figure 2. Note that the extinction coefficient, $\kappa_{s}$, is directly proportional to the absorption coefficient of the layer and the two are related by the equation $\alpha_{s}=4 \pi \kappa_{s} / \lambda_{0}$. The graph shows sensitivity increasing with interaction length up to a critical interaction length, $L_{\mathrm{c}}$, after which it decreases exponentially. The value

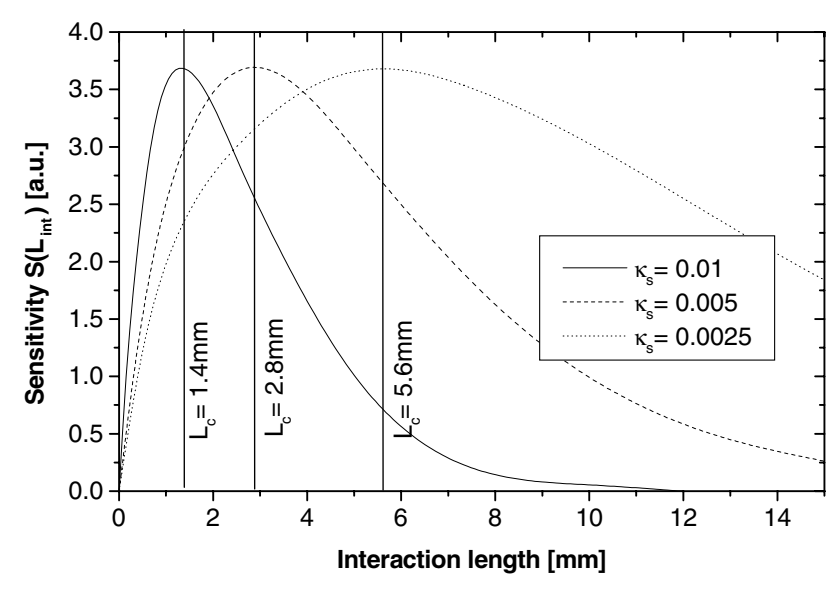

Figure 3. Predictions of theoretical model.

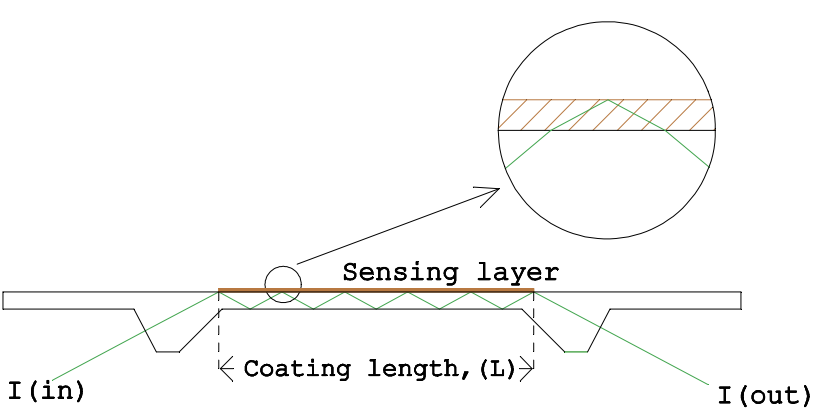

Figure 4. Schematic of polymer waveguide platform.

of $L_{\mathrm{c}}$ is dependent on the extinction coefficient of the sensing layer, with highly absorbing layers having the shortest $L_{\mathrm{c}}$. The obvious implication of this model is that increasing the number of reflections (i.e. using a longer waveguide) does not guarantee greater sensitivity when employing this optimized mode of interrogation. However, for a given absorption coefficient $\alpha_{s}$, there is an optimum interaction length, $L_{\mathrm{c}}$. It is clear then that there is a trade-off between $\alpha_{s}$ and $L_{\text {int }}$. We have investigated this trade-off experimentally in order to determine an optimized combination of the two and to establish a practical optimization strategy. The results of this investigation and their agreement with theoretical predictions are detailed in section 3.1.

\section{Experimental details}

\subsection{Design and fabrication of polymer waveguide platform}

One of the primary aims of this work was to develop massproducible, disposable platforms that exploit the possible sensitivity enhancement provided by a multiple reflection configuration. It was decided to avail of polymer processing technology in order to produce the sensing platforms, because of the wide range of low-cost, optical polymers available along with several manufacturing technologies which could be employed to produce the desired chips.

A schematic of the design developed by us for waveguide platforms that facilitate the enhanced interrogation of a thin sensing layer is shown in figure 4. The most important features of this design are the two refractive optic elements 
Enhanced polymer waveguide platforms for absorption-based sensors

at either end which facilitate coupling of the light into and out of the waveguide. These couplers ensure that light incident perpendicularly on their angled faces interrogates the sensing layer at the desired optimum angle, the value of which is dependent on several parameters including the refractive indices of the waveguide, sensing layer and environment. It is important to note that the waveguide chip is a single unit and the refractive elements should not be confused with prism couplers. It is evident from the schematic that only the region of the platform between the refractive couplers is involved in sensor operation. The portions at either end of the platform are included for ease of handling and incorporation into a custom made sensor head (see section 2.3).

Injection moulding was the fabrication method chosen as it is a well established, high throughput technique for which there is a wide range of optical quality polymers available. For the purposes of injection moulding it is necessary to fabricate a mould of the required dimensions in order to produce the polymer waveguide chips. This mould was designed using a commercially available CAD/CAM software package (XCAD, X-NC, UK) and fabricated from brass using a CNC micromiller (CAT3D, Datron, UK). The brass piece was then polished to produce surfaces having a high quality optical finish. This step is essential for the production of low loss waveguide platforms as it determines the surface roughness of the moulded waveguides.

The polymer platforms were produced using a Babyplast micro-injection moulder (JET Industrial Services, UK). Molten polymer — in this case, polystyrene (PS)—was injected into the polished mould where it was allowed to cool and solidify before being ejected in the form of plastic waveguide chips. Injection moulding is a high throughput technique and it was possible to produce 100 chips in approximately $5 \mathrm{~min}$. All chips were cleaned in an air plasma in order to remove organic contaminants from the surface of the polymer and to render it hydrophilic, thus improving the adhesion and uniformity of the $\mathrm{pH}$ sensitive thin film, the preparation of which is described in section 2.2.

\subsection{Preparation of sensing layer}

The $\mathrm{pH}$ sensitive layer consisted of a sol-gel derived thin film doped with bromocresol purple (BCP), a $\mathrm{pH}$ indicator dye from the sulfonephthalein family. A typical sol was prepared by dissolving the required amount of BCP in ethanol and then adding $\mathrm{pH} 1 \mathrm{HCl}$. Tetraethoxysilane (TEOS) and methyltriethoxysilane (MTEOS) (molar ratio TEOS:MTEOS, 9:1) were then added whilst stirring. The inclusion of MTEOS, a hydrophobic ORMOSIL (ORganically MOdified SILicate) reduced leaching of the indicator dye from the sensing layer upon exposure to the $\mathrm{pH}$ buffer solutions. The resultant sol was allowed to stir for $1 \mathrm{~h}$ and then aged at room temperature for $24 \mathrm{~h}$ before deposition. Sensing layers of varying lengths were deposited on the chips by spin-coating having first masked off the required areas. The coated platforms were then dried at $70{ }^{\circ} \mathrm{C}$ for $2 \mathrm{~h}$.

Samples were then immersed in a $2 \times 10^{-5} \mathrm{M}$ solution of $\mathrm{NaOH}$ until use as this treatment was found to condition the sensing layer, thereby producing a significantly reduced sensor response time [23].

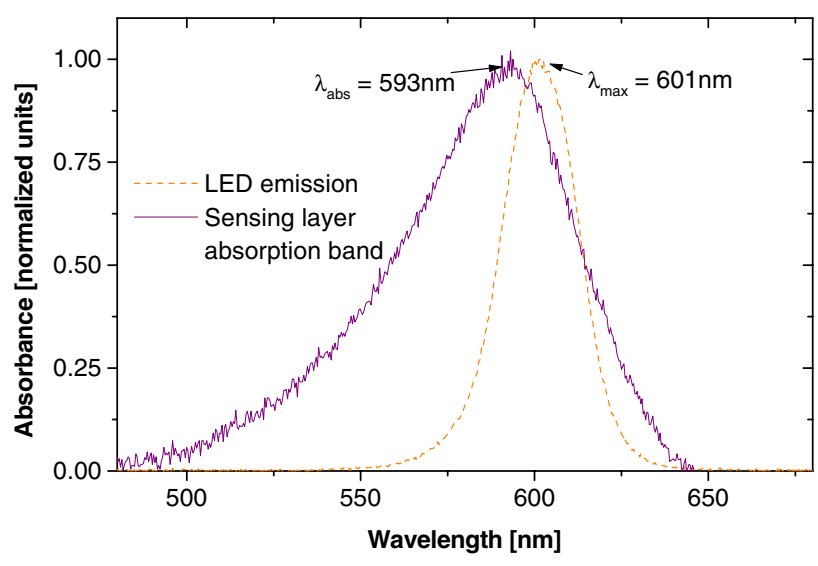

Figure 5. Absorption spectrum of sensing layer and LED emission spectrum.

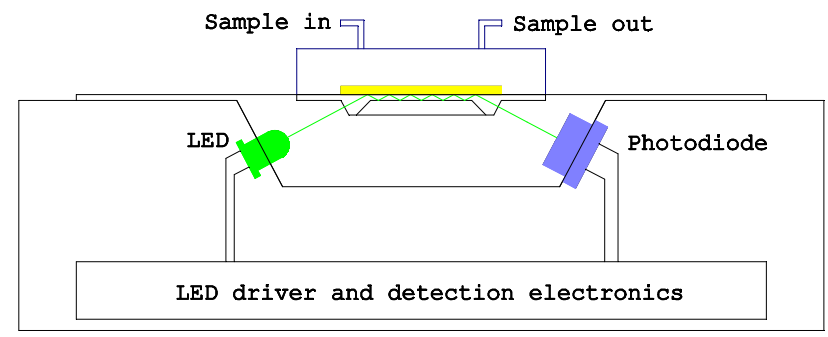

Figure 6. Sensor head design.

The absorption spectrum of the sensing layer was acquired using a Cary 50 Scan UV-vis spectrometer (Varian Inc., USA). This is shown in figure 5 along with the emission spectrum of the amber LED (Marl Optosource, UK) employed in the sensor head. The LED emission spectrum was taken using a S2000 microspectrometer (Ocean Optics Inc., USA). The absorption band of the deprotonated layer displays a maximum absorbance at $\lambda_{\mathrm{abs}}=593 \mathrm{~nm}$ which matches well with the LED emission $\left(\lambda_{\max }=601 \mathrm{~nm}\right)$. The large overlap between the two spectra demonstrates the suitability of the LED as a light source for this application.

\subsection{Operation of sensor system}

The coated platforms were placed in a custom-made aluminium head (see figure 6) which facilitated the coupling of the light from the LED source into the waveguide at the desired angle of incidence. The light was outcoupled via the second refractive element to a silicon photodiode detector. It is important to note that both the LED and photodiode were held in fixed positions relative to the polymer platform and this position was dictated by the theoretical model. The angular divergence of the LED was limited by an aperture in the sensor head to approximately $6^{\circ}$. A poly(dimethylsiloxane) (PDMS) flowcell connected to a peristaltic pump was used to pass $\mathrm{pH}$ buffer solutions over the sensing layer. The optoelectronic components were connected to a circuit which modulated the signal of the LED using an ICM555 timer chip. The modulation frequency served as the reference for an AD630 chip operating in lock-in mode which facilitated lock-in detection of the outcoupled light intensity by the photodiode. This signal was then recorded on a PC using 


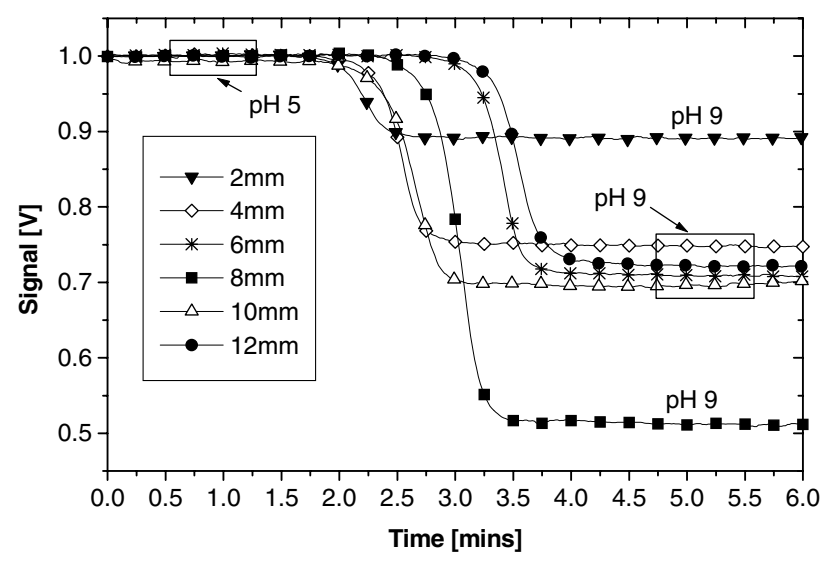

Figure 7. Sensor response for various coating lengths.

Table 1. Recorded signal change for each coating length.

\begin{tabular}{ll}
\hline Coating length $(\mathrm{mm})$ & Signal change $(\mathrm{V})$ \\
\hline 2 & 0.109 \\
4 & 0.252 \\
6 & 0.291 \\
$* 8$ & 0.487 \\
10 & 0.298 \\
12 & 0.277 \\
\hline
\end{tabular}

LabVIEW software which plotted the signal intensity as a function of time.

\section{Results and discussion}

\subsection{Sensor response for varying interaction lengths}

Sensing layers of varying length were deposited on the platforms by spin coating. All layers were examined by profilometry to ensure that their thicknesses were approximately $800 \mathrm{~nm}( \pm 50 \mathrm{~nm})$. This is an important aspect of sensor design due to the fact that the angle of optimum interrogation is dependent not only on the relevant refractive indices but also on the thickness of the sensing layer. In this case, the optimum angle of incidence was calculated for a layer thickness of $800 \mathrm{~nm}$. A variation in thickness of $100 \mathrm{~nm}$ results in a difference of less than $1^{\circ}$ in $\theta_{\max }$. Taking into account the limited angular divergence of the LED (as described in the previous section) a variation of $1^{\circ}$ in optimum angle would not unduly affect sensor performance.

Figure 7 illustrates the response of sensing platforms with a range of coating lengths to buffer solutions of $\mathrm{pH} 5$ and $\mathrm{pH} 9$, respectively. The higher voltage is the value obtained at pH5. The maximum voltage was offset to $1 \mathrm{~V}$ when plotting the data to aid in the comparison of signal change for each sample. The coating lengths for these platforms varied over the range $2 \mathrm{~mm}$ to $12 \mathrm{~mm}$. It should be noted that the response profiles have been offset with respect to one another on the $x$-axis in order to aid in their visualization and that the sample injection and response times did not vary significantly from sample to sample. Table 1 shows the signal change in volts for each of the coating lengths studied. The maximum signal changeand hence maximum sensitivity - was observed for a coating length of $8 \mathrm{~mm}$ with greater lengths resulting in a reduction in

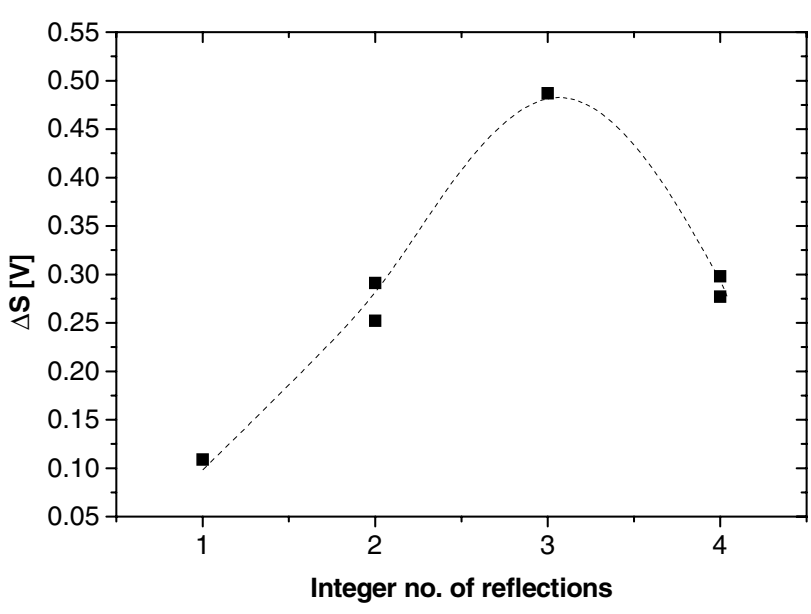

Figure 8. Dependence of signal change (related to sensor sensitivity) on integer number of reflections.

signal change. These findings are in qualitative agreement with theoretical predictions and clearly demonstrate the existence of an optimum length, $L_{\mathfrak{c}}$, for which sensitivity is maximum and, furthermore, that this length does not correspond to the maximum coating length studied.

It is important here to distinguish between coating length and optical interaction length. The theoretical predictions are based on the assumption that the incident light interrogates the entire length of the sensing layer for each interaction length investigated. However, the multimode nature of the waveguide platforms employed in this work results in the light interrogating the sensing layer in the form of discrete reflections as it propagates along the waveguide. The actual optical interaction length is directly proportional to the number of reflections from the sensing layer. With this in mind, the number of such discrete reflections was calculated for each coating length used in our experiments. The results of this analysis are shown in figure 8 where the existence of a clear peak in sensitivity is evident. This is entirely in keeping with the predictions of the theoretical model as illustrated in figure 3. It must be noted that this approach is empirical in nature as it is not possible to ascertain the actual interaction length of each sample due to difficulties in determining the extent of each reflection in the sensing layer. For this reason, although the results cannot be compared directly to theoretical predictions, they do serve to highlight the existence of the peak in sensitivity that is predicted by the model.

This peak in sensitivity has major implications for sensor design as it enables selection of the optimum platform dimensions. The platform can therefore be tailored to specific applications as the concentration ranges of interest, together with the specific dye used, determine the values of $\alpha_{s}$, and hence the optimum interaction length required. The semiempirical optimization strategy therefore consists initially of identification of the optimum number of reflections on a platform designed to operate at $\theta_{\text {opt. }}$. For example, in the data presented in figure 8 , the optimum number of reflections is three and the platform dimensions and coating length can then be adjusted accordingly. 


\section{Conclusions}

This work has described the development of mass-producible, enhanced polymer waveguide platforms. The theoretical analysis developed previously and applied to a single reflection platform was extended to a multiple reflection configuration in order to investigate the dependence of sensitivity on interaction length. The result of this analysis was that, for a given absorption coefficient of the sensing layer, there existed a peak in sensitivity that was achieved for a certain critical interaction length.

These results were investigated experimentally and signal change was plotted as a function of number of reflections interacting with each sensor platform. A peak in sensitivity for a critical number of reflections was evident. While this analysis was empirical in nature it confirms the existence of optimum sensing conditions in a multiple reflection configuration as predicted by the model. These results have significant implications for the design of miniaturized, yet highly sensitive polymer sensor platforms. As a figure of merit, the $\mathrm{pH}$ resolution yielded by these chips $(0.036 \mathrm{pH}$ units) is comparable to that of a previously developed fibrebased system with an interaction length of over seven times that of the coating length of the waveguides presented here [24]. The dependence of sensitivity on interaction length can also be exploited to tune the sensitivity of the platform to certain concentration ranges (i.e. certain absorption coefficients) in particular applications.

The results obtained show clear evidence of a critical interaction length for which sensitivity is maximized, a feature that is in agreement with theoretical predictions. This strategy shows great potential for the fabrication of lowcost, disposable optical absorption-based sensor platforms that exhibit enhanced sensitivity compared to current commonly employed configurations.

\section{References}

[1] Klein R and Voges E 1993 Integrated-optic ammonia sensor Sensors Actuators B 11221

[2] Grady T, Butler T, MacCraith B D, Diamond D and McKervey M A 1997 Optical sensor for gaseous ammonia with tuneable sensitivity Analyst $\mathbf{8} 803$

[3] Trinkel M, Trettnak W, Reininger F, Benes R, O'Leary P and Wolfbeis O S 1996 Study of the performance of an optochemical sensor for ammonia Anal. Chim. Acta 320235

[4] Werner T, Klimant I and Wolfbeis O S 1995 Ammoniasensitive polymer matrix employing immobilized indicator ion pairs Analyst 1201627

[5] McMurray H N 1992 Novel thin optical film sensors for the detection of carbon dioxide J. Mater. Chem. 2401
[6] Weigl B H, Holobar A, Rodriguez N V and Wolfbeis O S 1993 Chemically and mechanically resistant carbon dioxide optrode based on a covalently immobilized $\mathrm{pH}$ indicator Anal. Chim. Acta 282335

[7] Mills A and Chang Q 1994 Tuning colourimetric and fluorimetric gas sensors for carbon dioxide Anal. Chim. Acta 285113

[8] Piraud C, Mwarania E, Wylangowski G, Wilkinson J, O’Dwyer K and Schiffrin D J 1992 Optoelectrochemical thin-film chlorine sensor employing evanescent fields on planar optical wave-guides Anal. Chem. 64651

[9] Skrdla P J, Saavedra S S, Armstrong N R, Mendes S B and Peyghambarian N 1999 Sol-gel based planar waveguide sensor for water vapour Anal. Chem. 711332

[10] Skrdla P J, Armstrong N R and Saavedra S S 2002 Starch-iodine films respond to water vapor Anal. Chim. Acta $\mathbf{4 5 5} 49$

[11] Somani P R, Viswanath A K, Aiyer R C and Radhakrishnan S 2001 Novel dye+solid polymer electrolyte material for optical humidity sensing Org. Electron. 283

[12] Yang L and Saavedra S S 1995 Chemical sensing using sol-gel derived planar waveguides and indicator phases Anal. Chem. 671307

[13] Jin Z, Su Y and Duan Y 2000 An improved optical pH sensor based on polyaniline Sensors Actuators B 71118

[14] Lin J and Liu D 2000 An optical pH sensor with a linear response over a broad range Anal. Chim. Acta 40849

[15] Villegas M A and Pascual L 1999 Sol-gel silica coatings doped with a $\mathrm{pH}$-sensitive chromophore Thin Solid Films 351103

[16] Sadaoka Y, Matsuguchi M, Sakai Y and Murata Y 1992 Optical humidity sensing characteristics of Nafion-dyes composite thin films Sensors Actuators B 7443

[17] Matias F A A, Vila M M D C and Tubino M 2003 A simple device for quantitative colorimetric diffuse reflectance measurements Sensors Actuators B 8860

[18] Jeevarajan A S, Vani S, Taylor T D and Anderson M M 2002 Continuous $\mathrm{pH}$ monitoring in a perfused bioreactor system using an optical pH sensor Biotechnol. Bioeng. 78467

[19] Hisamoto H and Suzuki K 1999 Ion-selective optodes: current developments and future prospects Trends Anal. Chem. 18513

[20] Puyol M, De Valle M, Garces I, Villuendas F, Dominguez C and Alonso J 1999 Integrated waveguide absorbance optode for chemical sensing Anal. Chem. 715037

[21] Polerecky L, Burke C S and MacCraith B D 2002 Optimization of absorption-based optical chemical sensors that employ a single-reflection configuration Appl. Opt. 412879

[22] Polerecky L 2002 Optimisation of multimode waveguide platforms for optical chemical sensors and biosensors $P h D$ Thesis Dublin City University

[23] Ismail F, Malins C and Goddard N J 2002 Alkali treatment of dye-doped sol-gel glass films for rapid optical $\mathrm{pH}$ sensing Analyst 127253

[24] Butler T M, MacCraith B D and McDonagh C M 1995 Development of an extended range fiber optic $\mathrm{pH}$ sensor using evanescent wave absorption of sol-gel entrapped $\mathrm{pH}$ indicators Chemical Biochemical, and Environmental Fiber Sensors VII, Proc. SPIE 2508 168-78 